\title{
ESTABILIDADE DE VITAMINAS NO PROCESSAMENTO DE ALIMENTOS: UMA REVISÃO
}

\author{
RENATA LABRONICI BERTIN ${ }^{1}$ \\ MAYARA SCHULZ ${ }^{2}$ \\ EDNA REGINA AMANTE ${ }^{3}$
}

\begin{abstract}
Com o aumento da disponibilidade e da necessidade de alimentos submetidos ao processamento industrial, aumenta a preocupação com a perda de vitaminas durante o processo de fabricação dos mesmos. As vitaminas podem ser influenciadas pelos efeitos do processamento, sendo que as perdas variam muito de acordo com as técnicas empregadas e o tipo de alimento. O objetivo do estudo foi realizar uma revisão bibliográfica sobre a estabilidade de vitaminas durante o processamento dos alimentos. Os estudos analisados foram selecionados em bases eletrônicas de dados publicados no período de 2003 a 2014. Os resultados apontam que as perdas de vitaminas nos alimentos processados ocorrem em menor ou maior intensidade de acordo com a natureza química dos alimentos e também pelo armazenamento e processamento adotados. Podese concluir que são crescentes os estudos para minimizar a degradação e aumentar a estabilidade das vitaminas nos alimentos, com a utilização de técnicas que resultem nas menores perdas e aplicação de novas tecnologias, visando o melhoramento nutricional dos mesmos.
\end{abstract}

1 Nutricionista, mestrado em Nutrição e doutorado em Ciência dos Alimentos pela Universidade Federal de Santa Catarina, professora do Departamento de Nutrição da Universidade Federal do Piauí. rlbertin@ yahoo.com

2 Nutricionista, mestranda em Ciências dos Alimentos pela Universidade Federal de Santa Catarina. schulzmay@gmail.com

3 Graduada em Química Industrial, mestrado em Ciência e Tecnologia de Alimentos pela Universidade Federal de Viçosa, doutorado em Engenharia de Produção pela Universidade Federal de Santa Catarina, professora associada 4 do Departamento de Ciência e Tecnologia de Alimentos da Universidade Federal de Santa Catarina. eamante_1957@yahoo.com.br 


\section{INTRODUÇÃO}

As vitaminas constituem um grupo heterogêneo de substâncias e nutrientes vitais, as quais desempenham funções específicas e individuais relacionadas ao crescimento, reprodução e manutenção da saúde. A ausência sistemática na dieta resulta, quase sempre, em crescimento e desenvolvimento deficientes e outras perturbações orgânicas, configurando-se um quadro sintomatológico característico de carências nutricionais (GREGORY, 2010).

Tradicionalmente as vitaminas são divididas em dois grupos com base em sua solubilidade e de acordo com as propriedades fisiológicas e físico-químicas comuns, podendo ser classificadas em lipossolúveis ( $A, D, E$ e $K$ ) e hidrossolúveis ( $C$ e complexo $B)^{1,2}$. Pelo fato de não serem sintetizadas pelo organismo humano em quantidade suficiente para o desempenho normal de suas funções, torna-se necessária à ingestão das vitaminas através dos alimentos (FRANCO, 2008; LEHNINGER, NELSON e COX, 2011).

O teor de vitaminas nos alimentos é bastante variado e fatores como a forma química e o estado físico no qual as vitaminas se encontram afetam diretamente sua absorção pelo organismo. Além disso, por serem compostos bastante sensíveis, podem ser degradados pela temperatura, presença de oxigênio, luz, umidade e pH (LESKOVÁ et al., 2006; FRANCO, 2008).

Em contrapartida, para assegurar que os produtos alimentícios mantenham sua qualidade por um período de tempo maior, a partir da inibição das reações de deterioração e ao mesmo tempo garantindo ao produto características desejáveis do ponto de vista sensorial, são empregadas algumas técnicas de processamento, como refrigeração, congelamento, desidratação, salga, adição de açúcar, acidificação, fermentação, pasteurização, esterilização, utilização de pulsos elétricos, tecnologia de barreiras ou métodos combinados, entre outros (FELLOWS, 2006; PEREIRA e VICENTE, 2010).

Neste contexto, com o aumento da disponibilidade e da necessidade de alimentos submetidos ao processamento industrial, aumenta a preocupação com a perda de nutrientes durante o processo de fabricação dos mesmos, principalmente no que diz respeito às perdas de vitaminas (RIAZ, ASIF e ALI, 2009) gerando uma análise comparativa entre os alimentos ditos não processados, com reduzida vida de prateleira e sujeitos a inúmeras alterações, com os alimentos processados ou protegidos contra estas alterações.

Informações relacionadas à estabilidade das vitaminas nos alimentos estão disponíveis, porém constumam ser insuficientes, pois existe um entendimento limitado sobre os mecanismos de reação, cinética e termodinâmica sob condições diversas (GREGORY, 2010).

Desta forma, considerando os aspectos comparativos entre o alimento não processado e o alimento processado em relação às alterações na composição qualitativa e quantitativa das vitaminas, bem como, a disponibilidade crescente de alimentos submetidos ao processamento industrial nos últimos anos, este estudo tem por objetivo realizar uma revisão bibliográfica sobre os efeitos do processamento industrial dos alimentos sobre a estabilidade de vitaminas.

\section{MATERIAIS E MÉTODOS}

Realizou-se um levantamento bibliográfico em bases de dados eletrônicas com o objetivo de identificar estudos nacionais e internacionais, publicados no período de 2003 a 2014, que avaliaram a estabilidade das vitaminas no processamento de alimentos.

As bases eletrônicas consultadas foram a Scientific Eletronic Library On-line (SciELO), Science Direct, Scopus e o Portal Periódicos Capes. Os termos utilizados foram, vitaminas, estabilidade, processamento de alimentos e seus correspondentes em inglês.

Os artigos selecionados foram analisados em relação ao tipo de vitamina estudada, às características da amostra e ao tipo de processamento empregado. 
A Tabela 1 apresenta os artigos segundo autoria e ano de publicação, tipo de vitamina, alimento e tratamento aplicado. Também foram utilizados como fontes de pesquisa livros da área de Nutrição e Tecnologia de Alimentos considerados relevantes para a realização dessa revisão. Por fim, realizou-se a análise descritiva da amostra bibliográfica, acompanhada de discussão sobre os aspectos abordados.

\subsection{VITAMINAS HIDROSSOLÚVEIS}

\subsubsection{Vitamina C}

Dos estudos abordados, observou-se que muitos avaliaram a estabilidade da vitamina C. Sabe-se que a vitamina $C$ é uma das substâncias de grande significado para a nutrição humana, pois desempenha funções biológicas relacionadas ao sistema imune, formação de colágeno, absorção de ferro, inibição da formação de nitrosaminas e atividade antioxidante. O seu teor é influenciado pelo tipo de solo, forma de cultivo, condições climáticas, procedimentos agrícolas para colheita e armazenamento (COMBS, 2003; FRANCO, 2008). Além disso, o ácido ascórbico, forma reduzida da vitamina $\mathrm{C}$, é facilmente destruído por oxidação, particularmente na presença de calor, alcalinidade, catalisadores metálicos, danos físicos e baixa umidade relativa (GIANNAKOUROU e TAOUKIS, 2003), o que pode justificar o grande número de artigos que abordaram os fatores que interferem na estabilidade da vitamina $C$ no processamento de alimentos.

Em estudo realizado por Yamashita et al. (2003), os autores determinaram a estabilidade da vitamina $\mathrm{C}$ em acerola in natura e polpa pasteurizada, ambas congeladas a - $12 \mathrm{e}-18^{\circ} \mathrm{C}$, e de suco de acerola pasteurizado engarrafado, mantido à temperatura ambiente, ao longo de 4 meses de armazenagem. Os autores concluíram que a estabilidade de vitamina $\mathrm{C}$ em produtos de acerola foi dependente tanto do tipo de processamento quanto da temperatura de armazenagem, e os produtos que combinaram a pasteurização com congelamento apresentaram maior retenção de vitamina $\mathrm{C}$ ao final do período de armazenagem, independente da temperatura. Após quatro meses de armazenagem as acerolas in natura armazenadas a $-12 \mathrm{e}-18^{\circ} \mathrm{C}$ apresentaram, respectivamente, uma perda de $43 \%$ e $19 \%$. As taxas de degradação foram 2,4 vezes maiores a $-12{ }^{\circ} \mathrm{C}$ do que a -18 ${ }^{\circ} \mathrm{C}$. As amostras de polpa a $-12 \mathrm{e}-18^{\circ} \mathrm{C}$ apresentaram uma pequena perda, cerca de $3 \%$ após os quatros meses de armazenagem e o suco de acerola apresentou uma perda de $32 \%$ de vitamina $\mathrm{C}$.

Como observado, a estabilidade da vitamina $C$ é influenciada pelo aumento da temperatura e pelo aumento do tempo de armazenamento (LEE e KADER, 2000; MANSO et al., 2001).

As perdas de vitamina $C$ mesmo durante o congelamento podem ser explicadas pela atividade da enzima ascorbato oxidase, que mesmo a temperatura de $-18{ }^{\circ} \mathrm{C}$, se não inativada previamente, oxida eficientemente o ácido ascórbico (CARDELLO, MORAES e CARDELLO, 1994).

Este fato também pode ser observado em estudo realizado por Silva et al. (2004) que estudaram a estabilidade da vitamina $\mathrm{C}$ em pseudofruto de caju-do-cerrado, refrigerado $\left(4{ }^{\circ} \mathrm{C}\right) \mathrm{e}$ congelado $\left(-18^{\circ} \mathrm{C}\right.$ ), em diferentes tempos de armazenamento (30 e 90 dias) e embalagens (sacos de polietileno de baixa densidade e papel alumínio em sacos de polietileno). Os autores verificaram que os pseudofrutos refrigerados tiveram redução no conteúdo de ácido ascórbico comparado aos valores do pseudofruto in natura, além disso, houve redução gradativa durante o período de armazenamento dos pseudofrutos em embalagens opacas e transparentes. A redução do teor de ácido ascórbico no final do período de refrigeração alcançou valores aproximados de 84 e 92\%, nos pseudofrutos acondicionados em embalagens opaca e transparente, respectivamente. Os autores concluíram que o congelamento mostra-se mais eficiente do que a refrigeração na conservação dos teores de vitamina $\mathrm{C}$, entretanto o congelamento por um período superior a trinta dias reduz a sua estabilidade. Foi observado também que a embalagem opaca é mais efetiva na preservação dos teores de ácido ascórbico. 


\section{TABELA 1 RELAÇÃO DOS ESTUDOS SOBRE ESTABILIDADE DE VITAMINAS EM \\ ALIMENTOS PROCESSADOS DE 2003 A 2014 CONFORME AUTORIA E ANO DE PUBLICAÇÃO, TIPO DE VITAMINA, ALIMENTO E TRATAMENTO APLICADO.}

\begin{tabular}{|c|c|c|c|}
\hline Autor/ano & Tipo de Vitamina & Alimento & Tratamento \\
\hline $\begin{array}{l}\text { Yamashita et al, Brasil, } \\
2003\end{array}$ & Vitamina C & $\begin{array}{c}\text { Acerola in natura, polpa e suco } \\
\text { pasteurizados }\end{array}$ & Pasteurização e congelamento \\
\hline $\begin{array}{l}\text { Prodanov et al, } \\
\text { Espanha, } 2004\end{array}$ & $\begin{array}{c}\text { Tiamina, Riboflavina e } \\
\text { Niacina }\end{array}$ & Leguminosas & $\begin{array}{l}\text { Cozimento e imersão com } \\
\text { aditivos }\end{array}$ \\
\hline Silva et al, Brasil, 2004 & Vitamina C & $\begin{array}{l}\text { Pseudofruto de caju-do- } \\
\text { cerrado }\end{array}$ & $\begin{array}{c}\text { Refrigeração, congelamento } \\
\text { e embalagens }\end{array}$ \\
\hline $\begin{array}{l}\text { Garcia \& Penteado, } \\
\text { Brasil, } 2005\end{array}$ & Vitaminas A e E & Balas de gelatina & $\begin{array}{l}\text { Processo de fabricação e } \\
\text { armazenamento }\end{array}$ \\
\hline $\begin{array}{l}\text { Rinaldi et al, Brasil, } \\
2005\end{array}$ & Vitamina C & $\begin{array}{l}\text { Repolho minimamente } \\
\text { processado }\end{array}$ & Refrigeração e embalagens \\
\hline Vikram et al, India, 2005 & Vitamina C & Suco de laranja & $\begin{array}{l}\text { Aquecimento convencional, } \\
\text { ôhmico, infravermelho e micro- } \\
\text { ondas }\end{array}$ \\
\hline $\begin{array}{c}\text { Lebiedzin'ska } \\
\text { \& Szefer, Polônia, } 2006\end{array}$ & $\begin{array}{l}\text { Tiamina, Riboflavina, } \\
\text { Vitamina B6 e Niacina }\end{array}$ & Grãos, cereais e sementes & Tratamento térmico e polimento \\
\hline $\begin{array}{c}\text { Bernhardt \& } \\
\text { Schlich, Alemanha, } \\
2006\end{array}$ & $\begin{array}{l}\text { Vitamina all-trans- } \beta \text { - } \\
\text { caroteno e } \alpha \text { - tocoferol }\end{array}$ & Brócolis e pimenta vermelha & $\begin{array}{c}\text { Diferentes métodos de cocção: } \\
\text { forno a vapor }\left(100^{\circ} \mathrm{C}\right) \text {, forno a } \\
\text { vapor com pressão }\left(120^{\circ} \mathrm{C}\right) \\
\text { microondas }\end{array}$ \\
\hline $\begin{array}{l}\text { Freitas et al, } \\
\text { Brasil, } 2006\end{array}$ & Vitamina C & Suco tropical de acerola & $\begin{array}{l}\text { Processamento Hot Fill } \\
\text { (garrafas de vidro) e asséptico } \\
\text { (embalagens cartonadas) }\end{array}$ \\
\hline $\begin{array}{l}\text { Ortigues-Marty et al, } \\
\text { França, } 2006 \\
\text { Plaza et al,'Espanha, } \\
2006\end{array}$ & $\begin{array}{l}\text { Vitamina } B_{12} \\
\text { Vitamina } C\end{array}$ & $\begin{array}{l}\text { Carne } \\
\text { Suco de laranja }\end{array}$ & $\begin{array}{c}\text { Tratamento térmico } \\
\text { Alta pressão, campo elétrico } \\
\text { pulsado e pasteurização } \\
\text { Processo de fabricação e }\end{array}$ \\
\hline $\begin{array}{l}\text { Gutzeit et al, Alemanha, } \\
\qquad 2007\end{array}$ & Vitamina $\mathrm{K}_{1}$ & Suco e concentrado de Berries & $\begin{array}{l}\text { armazenamento (refrigeração e } \\
\text { temperatura ambiente) }\end{array}$ \\
\hline $\begin{array}{l}\text { Queiroz et al, Brasil, } \\
2008\end{array}$ & Vitamina C & Goiabas & $\begin{array}{l}\text { Desidratação por imersão- } \\
\text { impregnação e secagem } \\
\text { complementar por convecção }\end{array}$ \\
\hline $\begin{array}{l}\text { Bui \& Small, Austrália, } \\
2009\end{array}$ & Riboflavia & Macarrão do tipo noodles & Cocção e fortificação \\
\hline Alves et al, Brasil, 2010 & Vitamina C & $\begin{array}{l}\text { Manga minimamente } \\
\text { processada }\end{array}$ & Refrigeração \\
\hline $\begin{array}{l}\text { George et al, França, } \\
2011\end{array}$ & Carotenóides & $\begin{array}{l}\text { Tomates vermelhos e } \\
\text { amarelos }\end{array}$ & Pasteurização e liofilização \\
\hline $\begin{array}{l}\text { Oliveira et al, Brasil, } \\
2012\end{array}$ & Vitamina C & Suco de manga & $\begin{array}{l}\text { Tempos e temperaturas de } \\
\text { armazenamento }\end{array}$ \\
\hline $\begin{array}{l}\text { Gratacós-Cubarsí et al, } \\
\text { Espanha, } 2013\end{array}$ & Vitaminas B1, B2, B3 e B6 & Presunto & $\begin{array}{c}\text { Maturação em diferentes } \\
\text { períodos e concentrações de } \\
\text { sais nitrificantes }\end{array}$ \\
\hline $\begin{array}{l}\text { Kapusta-Duch et al., } \\
\text { Polonia, } 2013\end{array}$ & Betacaroteno & Couve de bruxelas & $\begin{array}{c}\text { Congelamento, branqueamento } \\
\text { e embalagens }\end{array}$ \\
\hline $\begin{array}{l}\text { Pereira et al, Brasil, } \\
2013\end{array}$ & Vitamina C & Suco de caju & $\begin{array}{c}\text { Fermentação, adição de açúcar } \\
\text { e refrigeração }\end{array}$ \\
\hline $\begin{array}{l}\text { Jakobsen \& Knuthsen, } \\
\text { Dinamarca, } 2014\end{array}$ & $\begin{array}{l}\text { Vitamina } D_{2}, D_{3} e \\
\text { 25-hidroxivitamina } D_{3}\end{array}$ & Ovos, margarina e pães & Tratamento térmico \\
\hline $\begin{array}{l}\text { Kaushik et al, India, } \\
2014\end{array}$ & Vitamina $\mathrm{D}_{2}$ & Leite & $\begin{array}{l}\text { Pasteurização, esterilização, } \\
\text { refrigeração e presença de luz no } \\
\text { armazenamento }\end{array}$ \\
\hline
\end{tabular}


Sabe-se que sucos processados têm vida útil extensa quando comparados com os sucos frescos. Entretanto, perdas de nutrientes como a vitamina $\mathrm{C}$ podem ocorrer em função das condições de armazenamento (TEIXEIRA e MONTEIRO, 2006). Neste contexto, estudo realizado por Freitas et al. (2006), o qual avaliou a estabilidade da vitamina $C$ do suco tropical de acerola adoçado, elaborado pelos processos Hot Fill (garrafas de vidro) e em envase asséptico (embalagens cartonadas) durante 350 dias de armazenamento em condições similares às de comercialização $\left(28^{\circ} \mathrm{C} \pm 2^{\circ} \mathrm{C}\right)$, verificaram ao final do período de armazenamento uma redução nos teores de vitamina $\mathrm{C}$ na ordem de $23,61 \%$ para o suco elaborado pelo processo Hot Fill e de 35,95\% para o processo asséptico. Os autores sugerem que as maiores perdas no teor de vitamina $C$ observada nas embalagens cartonadas, podem ser explicadas pela ocorrência de resíduos de peróxido de hidrogênio neste tipo de embalagem, bem como a sua permeabilidade ao oxigênio, além da possibilidade da regeneração de enzimas no suco.

Pereira et al. (2013) avaliaram a estabilidade da vitamina C em suco de caju fermentado com Lactobacillus casei. Amostras do suco adoçadas e não adoçadas foram estudadas durante o armazenamento sobre refrigeração $\left(4{ }^{\circ} \mathrm{C}\right)$ por 42 dias. Os autores observaram que o conteúdo de vitamina $C$ reduziu ao longo da estocagem, sendo que esta redução foi menos intensa no suco fermentado (19,17 e 21,49\%, com e sem a adição de açúcar, respectivamente) em comparação com a amostra controle não fermentada $(40,58 \%)$.

Considerando que a temperatura de armazenamento e a embalagem de produtos minimamente processados são os fatores mais importantes no retardamento de perdas nutricionais, Rinaldi et al. (2005) avaliaram as alterações nos teores de vitamina $C$ no repolho minimamente processado armazenado por 15 dias em temperaturas de 0,5 e $10^{\circ} \mathrm{C}$ acondicionado em bandejas de poliestireno expandido, revestidas com filme flexível de policloreto de vinila (PVC) e embalagens de tereftalato de polietileno (PET). Os autores observaram redução do teor de vitamina $\mathrm{C}$ em todas as embalagens e temperaturas estudadas, sendo que a embalagem de PVC apresentou menor redução de vitamina $C$ quando comparado a embalagem PET. Houve redução em torno de $43 \%$ na embalagem PET a $10^{\circ} \mathrm{C}$, enquanto que as temperaturas de 0 e $5^{\circ} \mathrm{C}$ não diferiram estatisticamente entre si.

Alves et al. (2010) estudaram mangas (Mangifera indica L.) minimamente processadas e armazenadas durante 10 dias em diferentes temperaturas $\left(0,6\right.$ e $\left.10^{\circ} \mathrm{C}\right)$. Os autores verificaram que a degradação de vitamina $C$ foi mais lenta a $0{ }^{\circ} \mathrm{C}$ e que houve redução de $10,5 \%$ e $27 \%$ no teor de ácido ascórbico entre os dias 0 e 10 na temperatura de 0 e $6{ }^{\circ} \mathrm{C}$, respectivamente. As frutas armazenadas a $12{ }^{\circ} \mathrm{C}$ apresentaram maior constante de velocidade de degradação, com uma redução no teor de ácido ascórbico de $49,9 \%$ no quarto dia de armazenamento, quando essas frutas apresentaram perda de firmeza tornando-se impróprias para o consumo.

Oliveira et al. (2012) também estudaram a influência da temperatura e do tempo de estocagem em amostras de suco de manga armazenado em garrafas PET adquirido comercialmente. De acordo com os autores, as amostras do produto foram armazenadas em estufas a 25,35 e $45{ }^{\circ} \mathrm{C}$ providas de iluminação (650 lux) 24 horas por dia, durante 120 dias. A concentração de vitamina $\mathrm{C}$ em sucos de manga armazenados a 25,35 e $45^{\circ} \mathrm{C}$ variou de 26,8 $\mathrm{mg} / 100 \mathrm{~mL}$ (concentração inicial) a $16,89\left(25^{\circ} \mathrm{C}\right), 10,34\left(35^{\circ} \mathrm{C}\right)$ e $9,85 \mathrm{mg} / 100 \mathrm{~mL}\left(45^{\circ} \mathrm{C}\right)$, no final dos respectivos dias de armazenamento, o que corresponde a uma perda de 37, 61,4 e 63,2\%, respectivamente.

A estabilidade da vitamina $\mathrm{C}$ em alimentos processados por tecnologias emergentes também têm sido estudada em comparação aos processos tradicionais. Plaza et al. (2006) estudaram a estabilidade da vitamina $C$ em suco de laranja processado por alta pressão, campo elétrico pulsado e pasteurização lenta logo após o tratamento e durante 40 dias de armazenamento refrigerado a $4{ }^{\circ} \mathrm{C}$. Logo após o tratamento, todos os sucos de laranja tratados apresentaram diminuição inferior a $8 \%$ do teor de vitamina C. No final do armazenamento, os sucos processados em alta pressão e pasteurização lenta apresentaram perdas de 14 e $18 \%$ 
de vitamina $\mathrm{C}$, respectivamente. $\mathrm{O}$ suco processado por campo elétrico pulsado apresentou as maiores perdas, $31,05 \%$.

A degradação de vitamina $C$ em suco de laranja também foi estudada por Vikram et al. (2005). Os autores compararam a estabilidade da vitamina C em suco tratado por aquecimento convencional, por infravermelho, por micro-ondas e por aquecimento ôhmico em diferentes temperaturas $\left(50,60,75\right.$ e $\left.90{ }^{\circ} \mathrm{C}\right)$ e tempos ( 0,5 a 15 minutos). $\mathrm{O}$ aquecimento ôhmico proporcionou menor degradação de ácido ascórbico em todas as temperaturas utilizadas, seguido pelo infravermelho e pelo aquecimento convencional. A maior degradação do ácido ascórbico ocorreu quando foi utilizado micro-ondas.

Queiroz et al. (2008) avaliaram as alterações nos teores de vitamina C em goiabas submetidas ao processo de desidratação por imersão-impregnação (DII) em soluções de sacarose e de açúcar invertido e secagem complementar por convecção. Além disso, também avaliaram o efeito da temperatura sobre a estabilidade do ácido ascórbico ao final de 60 dias de armazenamento. A DII com açúcar invertido sem diluição acarretou menor retenção de vitamina $\mathrm{C}$ ( $30 \%$ de perdas) que as soluções de sacarose ( $5 \%$ de perdas) e açúcar invertido a $41 \%$ (10\% de perdas). A secagem por convecção promoveu perdas significativas de vitamina $\mathrm{C}$ (de 32 a $68 \%$ ), em todos os tratamentos avaliados. No armazenamento a $7^{\circ} \mathrm{C}$, as amostras pré-tratadas com açúcar invertido sem diluição apresentaram maior percentual de redução de vitamina $C$ que aquelas pré-tratadas com sacarose ou com açúcar invertido a $41 \%$. A $25{ }^{\circ} \mathrm{C}$ todos os tratamentos se igualaram estatisticamente, com níveis de redução entre 70 a $86 \%$ nos teores de vitamina $C$.

O fato de as amostras tratadas com sacarose apresentarem menor percentual de perdas de vitamina $C$ pode ser explicado pela formação de uma camada de sacarose na superfície das amostras, evitando assim a entrada de oxigênio para o interior das células e a consequente oxidação do ácido ascórbico (GREGORY, 2010). E em relação ao açúcar invertido, o fato de o mesmo ter sido diluído pode ter provocado um menor potencial osmótico do que aquele realizado sem diluição, levando a um menor fluxo de água e de vitamina $\mathrm{C}$, do interior das amostras para o meio (QUEIROZ et al, 2008).

Frente ao exposto, os estudos sobre perdas de vitamina $\mathrm{C}$, necessitam ser avaliados comparativamente à disponibilidade de fontes frescas desta vitamina e a possibilidade de consumo; além disso, em alimentos processados, deve-se avaliar os processos que minimizam a lixiviação durante o pré-processamento, os efeitos das enzimas endógenas e exógenas, além das reações alimento - embalagem, condições do meio desde a colheita até a distribuição do produto final e o processamento propriamente dito, com vistas a disponibilidade de alimentos processados de alto valor nutricional e seguros ao consumidor.

\subsubsection{Vitaminas do complexo B}

As vitaminas do complexo B são um conjunto de vitaminas, que apesar de quimicamente diferentes, atuam no organismo de maneira muito semelhante. Dentro deste complexo estão as vitaminas: tiamina $\left(B_{1}\right)$, riboflavina $\left(B_{2}\right)$, piridoxina $\left(B_{6}\right)$, ácido pantotênico $\left(B_{5}\right)$, niacina $\left(B_{3}\right)$, biotina $\left(B_{7}\right.$ ou $\left.B_{8}\right)$, ácido fólico $\left(B_{9}\right)$ e cobalamina $\left(B_{12}\right)$. As mesmas estão relacionadas com inúmeras funções, como o metabolismo energético, o funcionamento do sistema nervoso e o metabolismo e equilíbrio de hormônios (COULTATE, 2004; FRANCO, 2008).

Por serem hidrossolúveis, as vitaminas do complexo B possuem maiores perdas no cozimento doméstico ou no processamento comercial de alimentos, entretanto o grau de degradação depende de como o alimento é cortado, enxaguado e do tempo de cocção ${ }^{24}$. Sabe-se que são mais estáveis em meio ácido e rapidamente degradadas em ambientes alcalinos. As taxas de degradação também aumentam quando as temperaturas são superiores a temperatura ambiente (GREGORY, 2010). Além disso, as perdas destas vitaminas são muito específicas e dependem do tipo de alimento. Nos vegetais, cada vitamina existe em diferentes formas, podendo estar ligadas 
na base celular por ligação covalente ou outras menos fortes, o que influencia na difusão da mesma para o meio de imersão e sua posterior perda (PRODANOV, SIERRA e VIDAL-VALVERDE, 2004).

A tiamina é uma das vitaminas do complexo $B$ mais instáveis. Resiste ao aquecimento apenas em $\mathrm{pH}$ menor do que 5,0. Ao contrário da tiamina, a riboflavina é uma das vitaminas mais estáveis, no entanto possui sensibilidade à luz. A piridoxina, a niacina, a cobalamina e o ácido fólico são facilmente degradados durante o processamento, por meio da lixiviação (COULTATE, 2004).

Lebiedzin'ska \& Szefer (2006) estudaram a estabilidade de vitaminas do complexo B (tiamina, riboflavina, piridoxina e niacina) em grãos e cereais in natura e seus produtos. Os resultados mostraram que os produtos naturais são uma fonte incomparavelmente melhor de vitaminas do complexo $\mathrm{B}$ do que os processados. Em relação à tiamina, os autores observaram que as concentrações diminuiram acentuadamente nos produtos processados, sendo menores no milho enlatado e maior no milho torrado e na farinha de milho. Já para a riboflavina, os conteúdos foram variáveis, sendo encontrado em maior concentração na farinha de trigo e cereais, e da mesma forma que na tiamina, o teor de riboflavina em produtos enlatados diminuiu cerca de $20 \%$. A piridoxina foi encontrada em níveis elevados em todos os alimentos analisados, e a maior concentração desta vitamina foi encontrada em farinhas de milho, milho torrado e grãos, e mais uma vez sua concentração em alimentos processados foi reduzida, principalmente nos enlatados. Em relação à niacina, as concentrações mais elevadas foram detectadas em sementes de gergelim, girassol e de abóbora, e nos demais produtos analisados, o teor de niacina foi bastante baixo.

Outro estudo, realizado por Prodanov et al (2004), avaliou os efeitos dos processos de cozimento e imersão e a influência de alguns aditivos (ácido cítrico e bicarbonato de sódio) no conteúdo de tiamina, riboflavina e niacina em grão de bico, feijões e lentilhas. Os autores verificaram que o feijão que permaneceu em imersão por nove horas teve perdas de 0 a $15 \%$ de tiamina, 0 a $11 \%$ de riboflavina e nenhuma alteração no conteúdo de niacina. O grão de bico teve perdas de 0 a $18 \%$ de tiamina, 0 a $4 \%$ de riboflavina e 0 a $46 \%$ de niacina. Em lentilhas, o processo de imersão diminuiu o conteúdo de tiamina e niacina de 5 a 10\% e 26 a 42\%, respectivamente e o conteúdo de riboflavina aumentou $98 \%$. Em relação à imersão com aditivos, em geral, as perdas de vitaminas foram maiores quando a imersão foi realizada em solução alcalina. No processo de cozimento, os autores verificaram que o mesmo não alterou o conteúdo de riboflavina, mas reduziu $35 \%$ de tiamina e $32 \%$ de niacina. O grão de bico e a lentilha tiveram maiores perdas, sendo que no grão de bico as perdas foram de até $51 \%$ de tiamina, $66 \%$ de riboflavina e $66 \%$ de niacina e na lentilha de até $78 \%$ de niacina e $61 \%$ de tiamina e riboflavina. O cozimento realizado após imersão prévia em solução alcalina causou maiores perdas das vitaminas.

Cabe destacar que outros fatores nutricionais necessitam ser considerados quando as leguminosas estão em discussão. Este grupo de alimentos, em geral, necessita de maceração visando contribuir para a eliminação de fatores antinutricionais como os taninos, ácido fítico, fito hemaglutininas dentre outros (GILANI, COCKELL e SEPEHR, 2005).

Ainfluência do $\mathrm{pH}$ e da temperatura sobre a degradação da riboflavina também foi observada por Bui e Small (2008), que avaliaram o impacto do processamento e da fortificação na retenção de riboflavina em três tipos de noodles asiáticos (branco salgado, amarelo alcalino e instantâneo). As amostras de macarrão foram cozidas, colocando-se uma pequena quantidade (aproximadamente 30 g) em uma panela de água fervente (600 mL), e após cada minuto de cocção um fio de macarrão era removido para posterior análise. Os resultados mostraram que houve uma considerável diferença no conteúdo de riboflavina para os diferentes estilos de macarrão e que a fortificação foi eficaz em melhorar o nível de vitamina total nos produtos após o cozimento. Além disso, verificou-se que ocorreram perdas em todas as etapas do processamento de todos os três estilos de noodles, sendo que as maiores perdas foram observadas em estágios no qual o aquecimento (na forma de vapor e fritura) estava envolvido, o que resultou em perdas de 52 a $74 \%$. O pH do produto também pareceu 
ser um fator de degradação para a riboflavina, visto que maiores perdas foram observadas no macarrão amarelo alcalino e instantâneo.

Gratacós-Cubarsí et al. (2013) estudaram o efeito da quantidade e das misturas de sais nitrificantes adicionados na maturação de presuntos sobre o teor das vitaminas $\mathrm{B}_{1}, \mathrm{~B}_{2}, \mathrm{~B}_{3}$ e $\mathrm{B}_{6}$. Foram adicionadas as seguintes concentrações de sais nitrificantes: $600 \mathrm{KNO}_{3} ; 150 \mathrm{KNO}_{3} ; 600 \mathrm{KNO}_{3}+600$ $\mathrm{NaNO}_{2} ; 150 \mathrm{KNO}_{3}+150 \mathrm{NaNO}_{2} ; 600 \mathrm{KNO}_{3}+600 \mathrm{NaNO}_{2}+500$ ascorbato de sódio (valores expressos em $\mathrm{mg}$ de sais adicionados na superfície por $\mathrm{kg}$ de presunto fresco). Os presuntos foram avaliados após 11,5 meses (processo padrão - PP) e 22 meses (processo longo - PL). Efeitos significativos dos diferentes tratamentos foram encontrados somente no teor de vitamina B6. Tanto em presuntos $\mathrm{PP}$, quanto em presuntos $\mathrm{PL}$, as amostras adicionadas apenas com nitrato apresentaram maior teor de vitamina B6 do que as amostras elaboradas com uma mistura de nitrato e nitrito. A adição de nitrito reduziu cerca de $40 \%$ do teor de vitamina $\mathrm{B}_{6}$. A adição de ascorbato de sódio no tratamento não afetou a concentração das vitaminas estudadas.

Ortigues-Marty et al. (2006) estudaram a influência do processamento térmico sobre o conteúdo de vitamina $B_{12}$ em carnes. Os processos de fritar e grelhar causaram um ligeiro aumento no teor de $B_{12}$ em comparação com a carne crua (de 1,5 a 2,4\%), entretanto, o processo de cozimento causou uma perda de 18,5 a 20,5\%. Sabe-se que a vitamina $B_{12}$ é relativamente mais resistente ao calor e que o tipo de tratamento térmico influencia no teor desta vitamina nos alimentos (GREGORY, 2010). A perda de vitamina $B_{12}$ pelo cozimento está relacionada com a perda de água durante este processo e pela destruição da vitamina que depende da temperatura e da duração da aplicação do calor (FELLOWS, 2006; GREGORY, 2010).

\subsection{VITAMINAS LIPOSSOLÚVEIS}

\subsubsection{Vitaminas A e E}

A vitamina A inclui o retinol e compostos relacionados, encontrados em tecidos animais, e alguns carotenoides, que são convertidos a retinol por enzimas da mucosa intestinal e estão presentes em alimentos tanto de origem animal, quanto vegetal (COULTATE, 2004; GREGORY, 2010).

A função fisiológica mais conhecida da vitamina A é o processo visual, no entanto, está envolvida também em atividades imunomoduladoras agindo como reguladora do crescimento e da diferenciação celular (YUYAMA et al, 2009; SOMMER e VYAS, 2012).

A degradação de retinoides e carotenoides com atividade pró-vitamina $A$ costuma ser paralela à degradação de lipídios insaturados (GREGORY, 2010). Quando em presença de oxigênio, os hidroperóxidos que resultam das reações de autoxidação dos ácidos graxos polinsaturados descolorem os carotenoides e retinoides. Os alimentos desidratados com uma grande área superficial exposta, em presença de oxigênio, possuem quebra rápida dos carotenoides (COULTATE, 2004).

Retinoides e carotenoides permanecem, na maioria das vezes, intactos durante o tratamento térmico, no entanto, podem sofrer isomerização. A exposição à luz durante o armazenamento pode exercer efeitos na retenção da vitamina $A$ em alimentos, visto que ocorrem reações de degradação fotoquímicas (GREGORY, 2010).

Com relação à vitamina $\mathrm{E}$, esta refere-se a uma família de compostos de ocorrência natural sintetizados pelas plantas, os tocoferois e os tocotrienois ${ }^{34}$, sendo os óleos de sementes de plantas as fontes mais importantes desses compostos na dieta. Outros tecidos vegetais e fontes animais possuem níveis baixos ${ }^{24}$. Também é conhecida como um antioxidante biológico, com função de proteger principalmente as estruturas lipídicas das membranas celulares dos efeitos nocivos dos radicais livres, desempenhando um importante papel na prevenção de doenças degenerativas (FRANCO, 2008; BOREL, PREVERAUD e DESMARCHELIER, 2013).

Os compostos vitamínicos $E$ apresentam certa estabilidade na ausência de oxigênio e assim 
como a vitamina A, a degradação é influenciada pelos mesmos fatores que influenciam a oxidação de ácidos graxos insaturado (GREGORY, 2010). Em óleos vegetais são bastante estáveis. Além disso, sua presença nos óleos impede o início da rancificação, mas inevitavelmente, a formação de peróxidos superará a presença de tocoferóis, o que causará sua oxidação. A atividade da vitamina E é rapidamente perdida em frituras (COULTATE, 2004).

A estabilidade das vitaminas A e E foi estudada por Garcia e Penteado (2005). Os autores verificaram a estabilidade das vitaminas em balas de gelatina no processo de fabricação e durante a vida de prateleira, variando-se a temperatura do processamento em 70 e $80^{\circ} \mathrm{C}$, e o efeito da ausência e presença do ácido cítrico. Os autores observaram que a vitamina que mais foi afetada pelo processamento das balas de gelatina foi à vitamina $\mathrm{A}$, perdendo em média $25 \%$ em relação ao adicionado, seguida da vitamina $E$ com uma perda média de $12 \%$. Em relação à temperatura, os autores observaram que aumentando a temperatura de 70 para $80^{\circ} \mathrm{C}$ foi encontrado uma redução da concentração média da vitamina $A$ de $37 \%$. Entretanto, para a vitamina $E$ não foram encontradas diferenças significativas. Em relação à adição do ácido cítrico, os autores observaram que foi benéfica para a estabilidade da vitamina $E$ em comparação à sua ausência, e os teores de vitamina $\mathrm{A}$ foram mais protegidos sem a adição do ácido cítrico. Durante a estocagem por seis meses, os autores verificaram uma perda média de $93 \%$ para a vitamina $A$ e $24 \%$ para a vitamina $E$.

Bernhardt \& Schlich (2006) avaliaram o impacto do cozimento sobre a retenção de vitaminas lipossolúveis (all-trans- $\beta$-caroteno e $\alpha$ - tocoferol), em brócolis e pimenta vermelha frescos e congelados. Os autores verificaram que nos brócolis frescos a cocção levou a uma redução significativa de all-trans- $\beta$-caroteno e $\alpha$-tocoferol (entre $14 \%$ e $25 \%$ ), enquanto que nos brócolis congelados nenhum decréscimo ocorreu. Já nas pimentas frescas e congeladas não foram observadas mudanças ou perdas significativas de $\alpha$-tocoferol e all-trans- $\beta$-caroteno. Os autores sugerem que a matriz alimentar, bem como a etapa de branqueamento aplicada nos vegetais congelados têm influência sobre a perda das vitaminas estudadas.

George et al. (2011) avaliaram o efeito da pasteurização e da liofilização sobre o teor de carotenoides em tomates vermelhos e amarelos. Os autores verificaram que a pasteurização não afetou o teor de carotenoides no tomate vermelho, mas diminuiu $44 \%$ do conteúdo de carotenóides no tomate amarelo. No processo de liofilização a redução do teor de carotenoides foi de $14 \%$ e $11 \%$ em tomates vermelhos e amarelos, respectivamente.

Kapusta-Duch et al. (2013) avaliaram a influência do processamento e do tipo de embalagem na estabilidade de $\beta$-caroteno em couve de bruxelas congelada. $O$ congelamento das amostras foi precedido pelos procedimentos de lavagem, branqueamento e resfriamento. Após, as amostras foram acondicionadas em sacos de polietileno e caixas plásticas de poliestireno e congeladas a $22{ }^{\circ} \mathrm{C}$ durante 3 meses, sendo que foram realizadas análises após 24 horas e 1, 2 e 3 meses. Os autores verificaram que o processo de branqueamento resultou na redução de $6,7 \%$ do teor de $\beta$-caroteno em comparação com vegetais crus. Já o processo de armazenamento por 24 horas não afetou o teor do componente estudado. Entretanto, após o primeiro mês de armazenamento, o conteúdo de $\beta$-caroteno reduziu significativamente, tanto em vegetais armazenados em sacos de polietileno e caixas poliestireno, sendo que as perdas foram de 7,1 e $9,7 \%$, respectivamente. Após o segundo mês de armazenamento, houve um declínio de 16,8\% nos sacos e 19,4\% nas caixas. Por fim, ocorreram perdas de $17,4 \%$ nos sacos e $19,6 \%$ nas caixas após os três meses de armazenamento.

\subsubsection{Vitamina $D$}

A vitamina $D$ pode ser encontrada na forma de colecalciferol (vitamina $D_{3}$ ), que está presente naturalmente nos alimentos, e como ergocalciferol (vitamina $D_{2}$ ), que é produzido sinteticamente, usado para aplicações farmacêuticas e suplementação alimentar (COULTATE, 
2004; FRANCO, 2008). Diversos metabólitos hidroxilados das vitaminas $D_{2}$ e $D_{3}$ são formados in vivo. O derivado 1,25-di-hidroxi de colecalciferol é a sua principal forma fisiologicamente ativa e o 25-hidroxicolecalciferol possui quantidade significativa de vitamina D de ocorrência natural em carnes e produtos lácteos (COMINETTI e COZZOLINO, 2009; GREGORY, 2010).

A principal função da vitamina $D$ em humanos é a manutenção das concentrações de cálcio e fósforo, a partir da regulação da absorção desses minerais no intestino e da atividade osteoblástica e osteoclástica dos ossos (FRANCO, 2008).

A vitamina $D$ é fotossensível e não se sabe se essa degradação é fotoquímica direta, por mecanismos que envolvem fotossensibilizadores que geram espécie reativa de oxigênio ou por efeitos indiretos da luz que levam à oxidação lipídica. Assim como outros compostos solúveis em lipídios, a vitamina D é sensível à degradação oxidativa (GREGORY, 2010).

A estabilidade da vitamina $D_{2}$ em leite fortificado foi estudada por Kaushik et al. (2014) que determinaram a perda desta vitamina durante o processamento, empacotamento e sob efeito da luz. O leite fortificado foi pasteurizado $\left(63^{\circ} \mathrm{C}, 30 \mathrm{~min}\right)$, fervido, esterilizado $\left(121^{\circ} \mathrm{C}, 15 \mathrm{~min}, 15 \mathrm{psi}\right)$, acondicionado em embalagens de vidro e de polietileno e armazenado a $4{ }^{\circ} \mathrm{C}$ durante sete dias. $\mathrm{A}$ estabilidade da vitamina $D_{2}$ no leite fortificado também foi estudada em três diferentes intensidades de luz (1485, 2970 e 4455 lux) após 2, 4, 8, 16 e 32 horas. Os autores verificaram que as perdas não foram significativas estatisticamente durante a pasteurização, fervura e esterilização. As perdas de vitamina $D_{2}$ também não foram significativas quando o leite foi armazenado durante sete dias em embalagens de vidro, entretanto quando armazenado em embalagens de polietileno, houve perda de 596,66 a 548,04 UI de vitamina $D_{2}$. Os autores também não verificaram perda significativa de vitamina $D_{2}$ quando as amostras foram armazenadas por 32 horas sob as três intensidades de luz em embalagens de vidro, mas verificaram perdas significativas nos leites armazenados em embalagens de polietileno após 4 horas de exposição nas três intensidades de luz, com perdas que variaram de 10,38 a $11,49 \%$.

Jakobsen e Knuthsenn (2014) investigaram a retenção de vitamina $D_{3}$ e 25-hidroxivitamina $D_{3}$ em ovos, vitamina $D_{3}$ na margarina, e vitamina $D_{3}$ e a vitamina $D_{2}$ em pães submetidos a tratamento térmico. Os autores verificaram que a retenção de compostos de vitamina $\mathrm{D}$ em ovos e margarina em forno durante 40 minutos à temperatura normal de cozimento mostraram retenção de 39 a $45 \%$, enquanto a fritura resultou na retenção de 82 a $84 \%$. Já os ovos cozidos tiveram um nível semelhante de retenção de compostos de vitamina D (86 a $88 \%$ ), não havendo diferença entre a retenção de vitamina $D_{3}$ e 25-hidroxivitamina $D_{3}$. A retenção de vitamina $D_{3}$ em pão de centeio foi de $69 \%$, mais baixa do que em pão de trigo, que foi $85 \%$. Uma retenção semelhante foi observada para a vitamina $\mathrm{D}_{2}, 73 \%$ e $89 \%$, respectivamente. Os autores concluíram que as perdas de vitamina $\mathrm{D}$ dependem do tipo de alimento e do processamento empregado. Desta forma, sugerem que deve-se otimizar os processos de cocção para se ter uma maior retenção desta vitamina.

\subsubsection{Vitamina $K$}

A vitamina $\mathrm{K}$ encontra-se em alimentos animais e vegetais, com a maior concentração em folhas verde escuras. A vitamina $\mathrm{K}$ se apresenta sob as formas de filoquinona (vitamina $\mathrm{K}_{1}$ ) que é a forma predominante, presente nos vegetais, sendo os óleos vegetais e as hortaliças suas fontes mais significativas; a dihidrofiloquinona (dK), formada durante a hidrogenação comercial de óleos vegetais; a menaquinona (vitamina $\mathrm{K}_{2}$ ), sintetizada por bactérias, presente em produtos animais e alimentos fermentados; e a menadiona (vitamina $\mathrm{K}_{3}$ ) que é um composto sintético convertido em $\mathrm{K}_{2}$ no intestino (KLACK e CARVALHO, 2006; MICHELAZZO e COZZOLINO, 2009). A vitamina $K$ é um componente essencial na coagulação sanguínea (COULTATE, 2004).

A vitamina $\mathrm{K}$ é bastante estável nas condições normais de processamento de alimentos e 
ao calor, porém pode sofrer degradação fotoquímica (GREGORY, 2010).

Gutzeit et al. (2007) investigaram o efeito do processamento de suco e concentrado de berries de Hippopha"e rhamnoides na degradação da filoquinona. A produção do suco industrial levou a uma perda de 36 a $54 \%$ de filoquinona. O processamento seguinte, que gerou o concentrado, resultou em uma depleção completa. Para determinar os efeitos da temperatura sobre a filoquinona durante o armazenamento o suco foi armazenado a 6,25 e $40^{\circ} \mathrm{C}$ durante até sete dias. A degradação foi de 18 a $32 \%$ nas três temperaturas estudadas, indicando que a intensidade de decomposição é independente da temperatura.

Como a vitamina $\mathrm{K}$ é estável ao calor, e a produção dos sucos teve impacto mínimo de luz, os autores acreditam que a degradação da vitamina foi devido ao baixo $\mathrm{pH}$ e ao uso de decantador para separar o sumo do bagaço, que levou também à perda da fração lipofílica dos frutos.

\title{
3 CONCLUSÃO
}

Diante do exposto, fica claro que diferentes vitaminas apresentam estabilidade variável, e que a perda é dependente da natureza química dos alimentos e do processamento aplicado.

Sabe-se que os vegetais e tecidos animais mantém suas atividades enzimáticas após a colheita ou abate, o que pode contribuir para as alterações nas concentrações de vitaminas dos alimentos. Essas alterações são inevitáveis, mas podem ser minimizadas quando procedimentos adequados referentes ao processamento, armazenamento e manipulação são adotados.

A constante busca por novos processos industriais têm como principal objetivo minimizar a degradação e aumentar a estabilidade das vitaminas nos alimentos submetidos a processamento industrial, através da utilização de técnicas que resultem nas menores perdas e aplicação de novas tecnologias, visando o melhoramento nutricional dos alimentos.

\section{STABILITY OF VITAMINS IN FOOD PROCESSING: A REVIEW}

\begin{abstract}
With the increased availability and necessity of food subjected to industrial processing, increases the concern about the loss of vitamins during the manufacturing process. The vitamins can be influenced by the effects of processing, while the losses vary widely according to the techniques of processing and type of food. The aim of the study was to review the literature on stability of vitamins in food processing. The studies analyzed were selected in bases of data electronics, published in the period from 2003 to 2014. The results indicate that losses of vitamins in processed foods found in greater or lesser intensity depending on the chemical nature of food and also for storage and processing adopted. It can be concluded that the studies to minimize the degradation and increase the stability of vitamins in food are increasing, new technologies can be applied and use techniques that result in lower losses in order to improve nutrition of the same.
\end{abstract}

KEY WORDS: VITAMINS, STABILITY, FOOD PROCESSING, FOOD 


\section{REFERÊNCIAS}

1 ALVES, J. Á.; NASSUR, R. C. M. R.; PIRES, C. R. F.; ALCÂNTARA, E. M.; GIANNONI, J. Á.; LIMA, L. C. O. Cinética de degradação de vitamina $c$ em mangas 'palmer' minimamente processadas armazenadas em diferentes temperaturas. Ciência e Agrotecnologia, v. 34, n. 3, p. 714-721, 2010.

2 ARRUDA, V. A. S.; PEREIRA, A. A. S.; ESTEVINHO, L. M.; ALMEIDA-MURADIAN, L. B. Presence and stability of B complex vitamins in bee pollen using different storage conditions. Food and Chemical Toxicology, v. 51, p. 143-148, 2013.

3 BERNHARDT, S.; SCHLICH, E. Impact of different cooking methods on food quality: Retention of lipophilic vitamins in fresh and frozen vegetables. Journal of Food Engineering, v. 77, p. 327-333, 2006.

4 BOREL, P.; PREVERAUD, D.; Desmarchelier, C. Bioavailability of vitamin E in humans: an update. Nutrition Reviews, v. 71, n. 6, p. $319-331,2013$.

5 BORTOLI, M. C.; COZZOLINO, S. M. F. Vitamina E (Tocoferol). In: COZZOLINO, S. M. F. Biodisponibilidade de nutrientes. 3. ed. Barueri: Manole, 2009.

6 BUI, L. T. T.; SMALL, D. M. Riboflavin in Asian noodles: The impact of processing, storage and the efficacy of fortification of three product styles. Food Chemistry, v. 114, p. 1477-1483, 2009.

7 CARDELLO, H. M. A. B.; MORAES, M. A. C.; CARDELLO, L. Ácido ascórbico e ascorbato oxidase em manga (Mangifera indica L.) var. Haden processada e congelada. Alimentos e Nutrição, v. 5, p. 65-75, 1994.

8 COMBS, J. R. Vitaminas. In: MAHAN, L. K.; ESCOTT-STUMP, S. (Eds.). KRAUSE: Alimentos, nutrição e dietoterapia. São Paulo: Ed. Rocca, 2003. p. 65-105.

9 COMINETTI, C.; COZZOLINO, S. M. F. Vitamina D (Calciferol). In: COZZOLINO, S. M. F. Biodisponibilidade de nutrientes. 3. ed. Barueri: Manole, 2009.

10 COULTATE, T. P. Alimentos: a química de seus componentes. 3. ed. Porto Alegre: Artmed, 2004. 368 p.

11 FELLOWS, P. Tecnologia do processamento de alimentos: princípios e prática. 2. ed. Porto Alegre, RS: ARTMED, 2006. 602p.

12 FRANCO, G. Tabela de composição química dos alimentos. 9a ed. São Paulo: Atheneu, c2008. 307p.

13 FREITAS, A. S.; MAIA, G. A.; COSTA, J. M. C.; FIGUEIREDO, R. W.; SOUSA, P. H. M.; FERNANDES, A. G. Estabilidade dos carotenoides, antocianinas e vitamina $C$ presentes no suco tropical de acerola (Malpighia emarginata dc.) adoçado envasado pelos processos hot-fill e asséptico. Ciência e Agrotecnologia, v. 30, n. 5, p. 942-949, 2006.

14 GARCIA, T.; PENTEADO, M. V. C. Qualidade de balas de gelatina fortificadas com vitaminas A, C e E. Ciência e Tecnologia de Alimentos, v. 25, n. 4, p. 743-749, 2005.

15 GEORGE, S.; TOURNIAIRE, F.; GAUTIER, H.; GOUPY, P.; ROCK, E. Changes in the contents of carotenoids, phenolic compounds and vitamin $C$ during technical processing and lyophilisation of red and yellow tomatoes. Food Chemistry, v. 124, p. 1603-1611, 2011.

16 GIANNAKOUROU, M. C.; TAOUKIS, P. S. Kinetic modelling of vitamin C loss in frozen green vegetables under variable storage conditions. Food Chemistry, v. 83, n. 1, p. 33-41, 2003.

17 GRATACÓS-CUBARSÍ, M.; SÁRRAGA, C.; CASTELLARI, M.; GUÀRDIA, M. D.; GARCÍA REGUEIRO, J. A.; ARNAU, J. Vitamin $\left(B_{1}, B_{2}, B_{3}\right.$ and $\left.B_{6}\right)$ content and oxidative stability of Gastrocnemius muscle from dry-cured hams elaborated with different nitrifying salt contents and by two ageing times. Meat Science, v. 95, p.647-651, 2013.

18 GREGORY, J. F. Vitaminas. In: DAMODARAN, S.; PARKING, K. L.; FENNEMA, O. R. Química de Alimentos de Fennema. $4^{a}$ ed. Editora: Artmed. Porto Alegre, 2010. 900 p.

19 GUTZEIT, D.; BALEABU, G.; WINTERHALTER, P.; JERZ, G. Determination of processing effects and of storage stability on vitamin K1 (Phylloquinone) in sea buckthorn berries (Hippopha"e rhamnoides L. ssp. rhamnoides) and related products. Journal of Food Science, v. 72, n. 9, p. 491-497, 2007.

21 KAPUSTA-DUCH, J.; BORCZAK, B.; KOPEC, A.; FILIPIAK-FLORKIEWICZ, A.; LESZCZYNSKA, T. The influence of packaging type and time of frozen storage on antioxidative properties of brussels sprouts. Journal of Food Processing and Preservation, p. 1- 8, 2013. 
22 KAUSHIK, R.; SACHDEVA, B.; ARORA, S. Vitamin D2 stability in milk during processing, packaging and storage. LWTFood Science and Technology, v. 56, p. 421-426, 2014.

23 KLACK, K; CARVALHO, J. F. Vitamina K: metabolismo, fontes e interação com o anticoagulante varfarina. Revista Brasileira de Reumatologia, v. 46, n. 6, p. 398-406, 2006.

24 LEBIEDZIN'SKA, A.; SZEFER, P. Vitamins B in grain and cereal-grain food, soy products and seeds. Food Chemistry, v. 95, p. 116-122, 2006.

25 LEE, S. K.; KADER, A. A. Preharvest and postharvest factors influencing vitamin $C$ content of horticultural crops. Postharvest Biology and Technology, v. 20, n. 3, p. 207-220, 2000.

26 LEHNINGER, A. L.; NELSON, D. L.; COX, M. M. Principios de bioquimica de Lehninger. 5. ed. Porto Alegre: ARTMED, 2011. 1273p.

27 LESKOVÁ, E.; KUBIKOVÁ, J.; KOVÁCIKOVÁ, E.; KOSICKÁ, M.; PORUBSKÁ, J.; HOLCIKOVÁ, K. Vitamin losses: Retention during heat treatment and continual changes expressed by mathematical models. Journal of Food Composition and Analysis, v. 19, p. 252-276, 2006.

28 MANSO, M. C.; OLIVEIRA, F. A. R.; OLIVEIRA, J. C.; FRÍAS, J. M. Modelling ascorbic acid thermal degradation and browning in orange juice under aerobic conditions. Int J Journal of Food Science and Technology, v. 36, p. 303-312, 2001.

29 MICHELAZZO, F. B.; COZZOLINO, S. M. F. Vitamina K. In: COZZOLINO, S. M. F. Biodisponibilidade de nutrientes. 3. ed. Barueri: Manole, 2009.

30 OLIVEIRA, A. N.; RAMOS, A. M.; MINIM, V. P. R.; CHAVES, J. B. P. Sensory stability of whole mango juice: influence of temperature and storage time. Food Science and Technology, v. 32, n. 4, 2012.

31 ORTIGUES-MARTY, I.; THOMAS, E.; PRÉVÉRAUD, D. P.; GIRARD, C. L.; BAUCHART, D.; DURAND, D. Influence of maturation and cooking treatments on the nutritional value of bovine meats: Water losses and Vitamin B12. Meat Science, v. 73, p. 451-458, 2006.

32 PEREIRA, A. L. F.; ALMEIDA, F. D. L.; JESUS, A. L. T.; COSTA, J. M. C.; RODRIGUES, S. Storage stability and acceptance of probiotic beverage from cashew apple juice. Food and Bioprocess Technology, v. 6, p. 3155-3165, 2003.

33 Pereira, R. N.; Vicente, A. A. Environmental impact of novel thermal and non-thermal technologies in food processing. Food Research International, v. 43, p. 1936-1943, 2010.

34 PLAZA, L.; SANCHEZ-MORENO, C.; ELEZ-MARTINEZ, P.; ANCOS, B.; MARTIN-BELLOSO, O.; CANO, M. P. Effect of refrigerated storage on vitamin $\mathrm{C}$ and antioxidant activity of orange juice processed by high-pressure or pulsed electric fields with regard to low pasteurization. European Food Research and Technology, v. 223, p. 223: 487-493, 2006.

35 PRODANOV, M.; SIERA, I.; VIDAL-VALVERDE, C. Influence of soaking and cooking on the thiamin, riboflavin andniacin contents of legumes. Food Chemistry, v. 84, p. 271-277, 2004.

36 GILANI, G. S.; COCKELL, K. C.; SEPEHR, E. Effects of antinutritional factors on protein digestibility and amino acid availability in foods. Journal of AOAC INTERNATIONAL, v. 88, n. 3, p. 967-87, 2005.

37 QUEIROZ, V. A. V.; BERBERT, P. A.; MOLINA, M. A. B.; GRAVINA, G. A.; QUEIROZ, L. R.; SILVA, J. A. Qualidade nutricional de goiabas submetidas aos processos de desidratação por imersão-impregnação e secagem complementar por convecção. Ciência e Tecnologia de Alimentos, v. 28, n. 2, p. 329-340, 2008.

38 RIAZ, M. N.; ASIF, M.; ALI, R. Stability of vitamins during extrusion. Critical Reviews in Food Science and Nutrition, v. 49 , n. $4,2009$.

39 RINALDI, M. M.; BENEDETTI, B. C.; CALORE, L. Efeito da embalagem e temperatura de armazenamento em repolho minimamente processado. Ciência e Tecnologia de Alimentos, v. 25, n. 3, p. 480-486, 2005.

40 SILVA, M. R.; SILVA, M. S.; OLIVEIRA, J. S. Estabilidade de ácido ascórbico em pseudofrutos de caju-do-cerrado, refrigerados e congelados. Pesquisa Agropecuária Tropical, v. 34, n. 1, p. 9-14, 2004.

41 SOMMER, A.; VYAS, K. S. A global clinical view on vitamin A and carotenoids. The American Journal of Clinical Nutrition, v. 96(suppl), p. 1204S-6S, 2006.

42 TEIXEIRA, M.; MONTEIRO, M. Degradação da vitamina C em suco de fruta. Alimentos e Nutrição, v. 17, n. 2, p. 219227, 2006. 
43 VIKRAM, V. B.; RAMESH, M. N.; PRAPULLA, S. G. Thermal degradation kinetics of nutrients in orange juice heated by electromagnetic and conventional methods. Journal of Food Engineering, v. 69, p. 31-40, 2005.

44 YAMASHITA, F.; BENASSI, M. T.; TONZAR, A. C.; MORIYA, S.; FERNANDES, J. G. Produtos de acerola: estudo da estabilidade de vitamina C. Ciência e Tecnologia de Alimentos, v. 23, n. 1, p. 92-94, 2003.

45 YUYAMA, L. K. O; MARINHO, H. Á.; ALENCAR, F. H.; YONEKURA, L.; COZZOLINO, S. M. F. Vitamina A (Retinol) e carotenóides. In: COZZOLINO, S. M. F. Biodisponibilidade de nutrientes. 3. ed. Barueri: Manole, 2009. 